\title{
Kinematics Analysis and Optimization of a Multi-Mode Mobile Parallel Mechanism
}

\author{
Chunyan ZHANG*, Mingjuan XIE, Dan ZHANG
}

\begin{abstract}
This paper aims to disclose the kinematics and optimize the design of multi-mode mobile parallel mechanism. To this end, a multi-mode mobile parallel mechanism was designed based on single-loop planar $4 \mathrm{R}$ systems. The horizontally symmetric mechanism is controlled by two motors: the mechanism can switch freely among different modes of movements (e.g. sliding, turning and rolling) by changing the input angles of the two motors. Based on the mechanism structure, the author analysed the degrees of freedom (DOFs) and kinematics of each mode, and optimized the stability of the mechanism. The results show that the mechanism is more stable at a short rod length and small rotating angle, when its width is constant. Finally, the theoretical correctness of the mechanism was verified through simulation and prototype test. The research findings provide a valuable reference for similar studies on multi-mode mobile parallel mechanism, and lay the theoretical basis for the application of mobile robots in various fields.
\end{abstract}

Keywords: degrees of freedom (DOFs); kinematics analysis; mobile parallel mechanism; single-loop planar 4R system

\section{INTRODUCTION}

Since its conception in 1978 [1], multi-mode parallel mechanism has undergone a profound evolution. For instance, Kong Xianwei [2-4] held that various spatial mechanisms of the same degree of freedom (DOF) can be constructed with spatial single-loop mechanisms in different geometric shapes. Miao Zhihuai [5], Tian Yaobin et al. [6] combined a multi-mode spatial mechanism and a mobile system into a multi-mode mobile mechanism, which realizes such motions as walking, creeping, selftraversing and rolling through modular selfreconfiguration. Mark Yim designed the PolyBot, a multimode mobile robot capable of snake-like creeping, circular scrolling and even ladder climbing. All these motions are achieved through modular self-reconfiguration [7]. Another famous multi-mode mobile robot, NASA's SuperBot also supports snake-like creeping, rolling and walking through self-reconfiguration [8].

In this paper, the single-loop planar $4 \mathrm{R}$ system was adopted as the basis of our multi-mode mobile parallel mechanism, considering the limited DOFs and simple control of single-loop closed-chain mechanism [3, 4, 9]. Then, the structural design method and motion principle of the said mechanism were investigated under each multimode, aiming to disclose the structural and motion features. The research findings provide a valuable reference to the design of mobile parallel mechanism [10].

\section{MECHANISM DESIGN AND DOF ANALYSIS \\ 2.1 Mechanism Design}

As shown in Fig. 1, the multi-mode mobile parallel mechanism consists of two 1DOF planar 4R systems (i.e. left foot and right foot), and a rod linking up the two $4 \mathrm{R}$ systems. Each foot has four rotation pairs $(R)$, whose axes are parallel to each other. The distance between two feet (i.e. the length of $R_{11} R_{21}$ ) is denoted as $p$, and the length of the remaining bars as $n$. Both lengths are measured in metres.

The multi-mode mobile parallel mechanism has the following features. (1) With two single-loop closed-chain planar 4R systems as the feet, our mechanism is bilaterally symmetrical, featuring $1 \mathrm{DOF}$ in each single-loop closed- chain. (2) The two 4R systems form a closed loop with the rod and the ground, which satisfies the structural requirements of the parallel mechanism. In sliding mode, the rod serves as a moving platform of the parallel mechanism and the ground as a static platform; in rolling mode, the rod, the left foot and the right foot contact with the ground in turns, and the dynamic and static platforms operate alternately, forming a multi-mode mobile parallel mechanism. (3) Our mechanism supports multi-mode motions like sliding, turning and rolling, as required for unstructured environment.

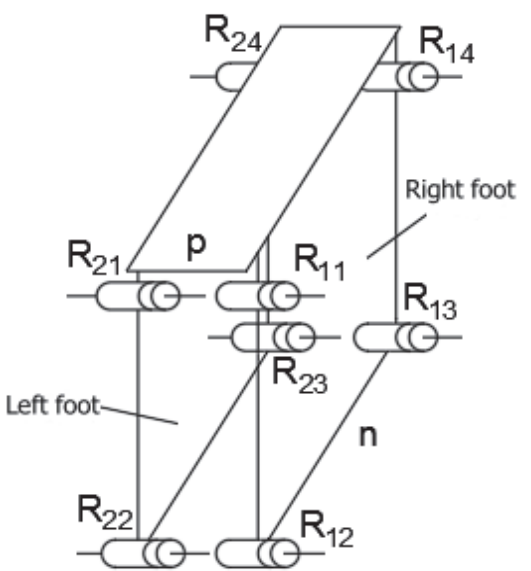

Figure 1 Sketch map of multi-mode mobile parallel mechanism

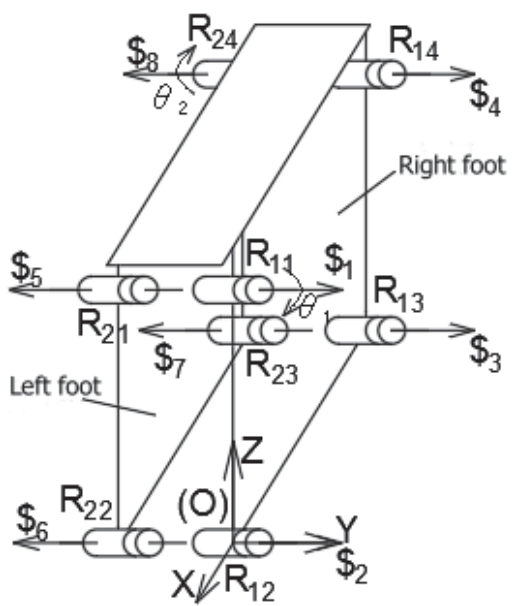

Figure $2 \mathrm{DOF}$ analysis of our mechanism in sliding mode 


\subsection{DOF Analysis of Sliding Mode}

Before the DOF analysis, a coordinate system was established for the multi-mode mobile parallel mechanism (Fig. 2). The kinematic helix and inverse helix of the mechanism were obtained by the screw theory [11], the DOF of our mechanism in the sliding mode can be expressed as 2 .

The mechanism has one rotational DOF around $Y$-axis and one moving DOF along $X$-axis. In light of the DOF analysis, the two diagonally distributed rotary pairs $R_{11}$ and $R_{24}$ were selected as the drive motors to control the mechanism, so that the sliding can be achieved through driving the left and right sides of the 4R system. Similarly, the turning can be achieved through the differential drive between the motors.

\subsection{DOF Analysis of Rolling Mode}

If the two motors in the mechanism have equivalent input angles but opposite directions, the left and right single-loops will share the same output angle; then, the mechanism will rotate synchronously, forming an integral parallel mechanism. In this way, the mechanism can enter the rolling mode.

Taking the left single-loop planar 4R mechanism as the object (Fig. 3), the author established a coordinate system to examine the DOF of the mechanism in rolling mode. The DOF of the mechanism in rolling mode can be obtained 1:

Thus, the mechanism has only $1 \mathrm{DOF}$ around $Y$-axis, and the driving effect of the two diagonally distributed motors is equivalent to that of a single motor.

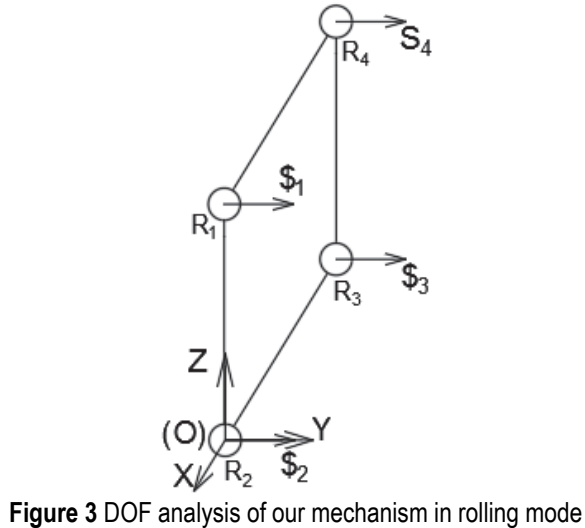

\section{KINEMATICS ANALYSIS}

3.1 Kinematics Analysis of Sliding Mode

3.1.1 Position Analysis of Sliding Mode

To obtain the direct kinematics solution to position, it is necessary to calculate the posture of the moving platform based on relevant joint variables. As shown in Fig. 4, the middle point of $R_{22} R_{23}$ was taken as the origin $\mathrm{O}$ of the fixed coordinate system; $A_{1}\left(x_{1}, y_{1}, z_{1}\right), A_{2}\left(x_{2}, y_{2}, z_{2}\right)$ and $A_{3}\left(x_{3}, y_{3}\right.$, $z_{3}$ ) are respectively the positions of the midpoints of $R_{21} R_{24}$, $R_{22} R_{23}$ and $R_{12} R_{13}$ in the fixed coordinate system, provided that the origin $\mathrm{O}$ coincides with the initial position of point $A_{2}$. Here, the midpoints of the left and right feet $A_{2}$ and $A_{3}$ are treated as the typical points, and Denavit-Hartenberg method [12] is adopted to derive the relationship between the input angles $\theta_{1}$ and $\theta_{2}$ and the outputs of the two feet.
When the rod $R_{23} R_{24}$ rotates by an angle of $\theta_{2}$, the position vector of point $A_{1}$ in the fixed coordinate system can be expressed as:

$$
\left[\begin{array}{l}
x_{1} \\
y_{1} \\
z_{1}
\end{array}\right]=\left[\begin{array}{c}
n \sin \theta_{2} \\
0 \\
n \cos \theta_{2}
\end{array}\right]
$$

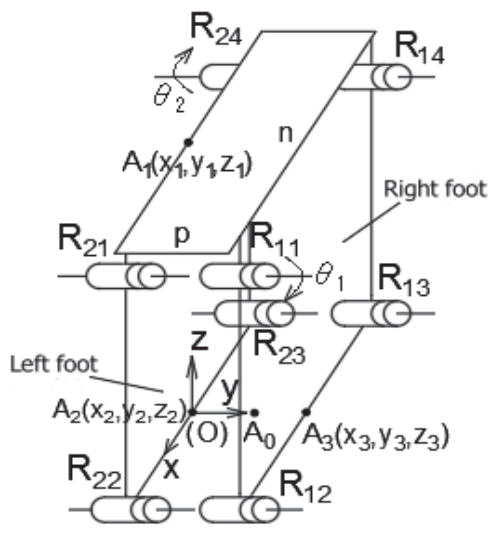

Figure 4 Position analysis in sliding mode

In this case, the displacement of the mechanism along the $Z$-axis induces the change to the centre of gravity of the mechanism. Similarly, points $A_{2}$ and $A_{3}$ are displaced by $y_{2}^{\prime}$ and $y_{3}^{\prime}$ in the $Y$-axis direction from the initial positions (Fig. 5). Using the geometric method, the calculation results can be modified as:

$$
\begin{aligned}
& y_{2}^{\prime}=\frac{n h}{p} \cos \theta_{2}+p-\sqrt{p^{2}-h^{2}} \\
& y_{3}^{\prime}=\frac{n h}{p} \cos \theta_{2}+p
\end{aligned}
$$

where $h\left(h=n\left(1-\cos \theta_{2}\right)\right)$ is the variation in $Z$-axis displacement of point $A_{1} ; G^{\prime}$ the centre of gravity of the mechanism; $v_{G}^{\prime}$ is the variation in $Y$-axis displacement of the centre of gravity of the mechanism.
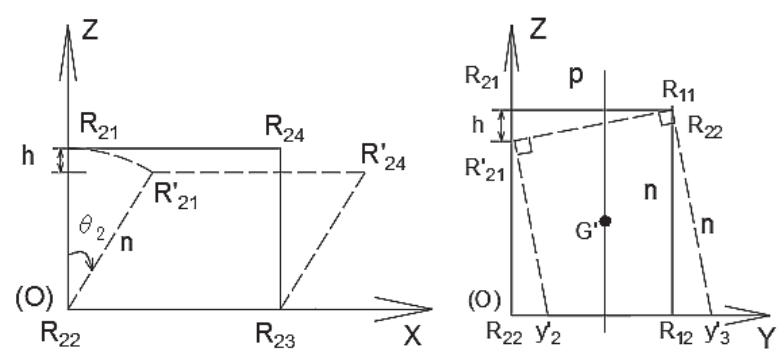

Figure 5 Position analysis diagram: (a) $h$; (b) $y_{2}^{\prime}$ and $y_{3}^{\prime}$

In sliding mode, the mechanism is always in contact with the ground. Thus, there is no displacement of the mechanism along the $z$-axis throughout the sliding. As shown in Fig. 4, when the rod $R_{23} R_{24}$ rotates by an angle of $\theta_{2}$, then, the position vectors of points $A_{2}^{\prime}$ and $A_{3}^{\prime}{ }_{3}$ in the fixed coordinate system can be expressed as: 


$$
\begin{aligned}
& {\left[\begin{array}{l}
x_{2}^{\prime} \\
y_{2}^{\prime} \\
z_{2}^{\prime}
\end{array}\right]=\left[\begin{array}{c}
n \sin \theta_{2} \\
p
\end{array}\right]+p-\sqrt{p^{2}-n^{2}\left(1-\cos \theta_{2}\right)^{2}}} \\
& 0 \\
& {\left[\begin{array}{l}
x_{3}^{\prime} \\
y_{3}^{\prime} \\
z_{3}^{\prime}
\end{array}\right]=\left[\begin{array}{c}
\frac{n^{2}\left(1-\cos \theta_{2}\right)}{p}+p \\
0
\end{array}\right]}
\end{aligned}
$$

where $A_{2}^{\prime}\left(x_{2}^{\prime}, y_{2}^{\prime}, z_{2}^{\prime}\right)$ and $A_{3}^{\prime}\left(x_{3}^{\prime}, y_{3}^{\prime}, z_{3}^{\prime}\right)$, are the positions of points $A_{2}$ and $A_{3}$ respectively rotate the angle of $\theta_{2}$ relative to the fixed coordinate system.

When the rod $R_{11} R_{12}$ rotates around the $Y$-axis by an angle of $\theta_{1}$, the position vectors of midpoints $A_{2}$ and $A_{3}$ in the fixed coordinate system can be expressed as:

$\left.\begin{array}{l}{\left[\begin{array}{l}x_{2} \\ y_{2} \\ z_{2}\end{array}\right]=\left[\begin{array}{c}n \sin \theta_{2} \\ p\end{array}\right]} \\ {\left[\begin{array}{l}x_{3} \\ y_{3} \\ z_{3}\end{array}\right]=\left[\frac{n \sin \theta_{1}}{p}-\sqrt{p^{2}-n^{2}\left(1-\cos \theta_{2}\right)^{2}}-\frac{n^{2}\left(1-\cos \theta_{1}\right)}{p}\right]} \\ 0\end{array}\right]$

According to Eqs. (2) and (3), the mechanism does not roll under the following condition:

$0 \leq y_{2}^{\prime} \leq y_{G}^{\prime} \leq y_{3}^{\prime} \leq p+\frac{2 n^{2}}{p}$

\subsubsection{Motion Space Analysis}

Considering the relationship between points $\left(A_{2}\right.$ and $\left.A_{3}\right)$ and input angles $\left(\theta_{1}\right.$ and $\left.\theta_{2}\right)$ and the stability requirements of the mechanism, the author simulated the mechanism in Matlab with $n=p=0.1 \mathrm{~m}$ and $\theta_{1}, \theta_{2}$ $\in\left(-\frac{\pi}{2}, \frac{\pi}{2}\right)$. Each variable was iterated 100,000 times.

Then, the periodical 3D motion space of the points $\left(A_{2}\right.$ and $\left.A_{3}\right)$ in the fixed coordinate system was calculated by Monte Carlo method [13] at input angles $\theta_{1}$ and $\theta_{2}$. It can be seen from Fig. 6 that the two feet were always symmetrical about the plane parallel to $X O Z$ during sliding. With the change of time, the left foot slid forward first towards the right front, and then the right foot slid forward towards left front. The two feet slid alternately until the completion of the periodic movement.
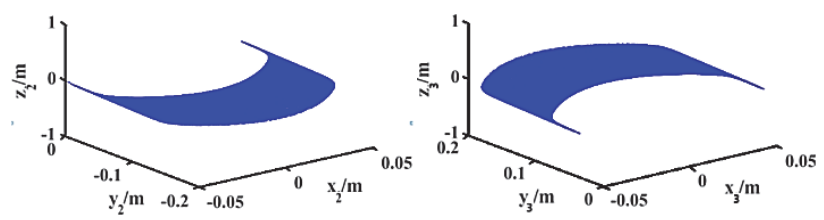

Figure 6 Periodical 3D motion space of points $A_{2}$ and $A_{3}$ : (a) Left foot; (b) Right foot.

\subsubsection{Motion Space of Line Sliding Mode}

As mentioned above, the left and right feet slid alternately towards the right and left fronts. Hence, when the input angles $\theta_{1}$ and $\theta_{2}$ satisfy $\theta_{1}, \theta_{2} \in\left(-\frac{\pi}{2}, \frac{\pi}{2}\right)$ the $X$ AXIS displacement of the mechanism equals the $Y$-axis displacement, which is approximately zero. At this time, the mechanism is switched to the straight line sliding mode (Fig. 7)

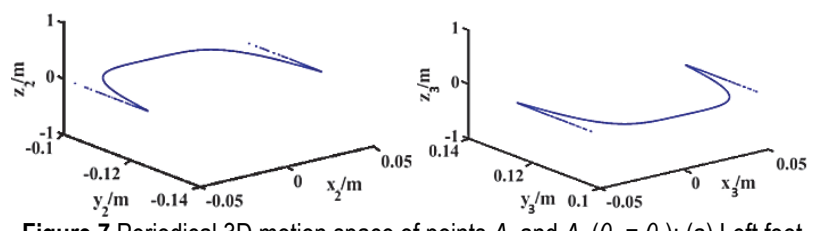

Figure 7 Periodical 3D motion space of points $A_{2}$ and $A_{3}\left(\theta_{1}=\theta_{2}\right)$ : (a) Left foot (b) Right foot

\subsubsection{Motion Space of Turning Sliding Mode}

When the input angles $\theta_{1}$ and $\theta_{2}$ meet $\theta_{1}>\theta_{2} \in\left(-\frac{\pi}{2}, \frac{\pi}{2}\right)$ the midpoints of the two feet $A_{2}$ and $A_{3}$ move at different step lengths along the $X$-axis and $Y$-axis. In positive direction of the $Z$-axis, the mechanism rotates clockwise around the $Z$-axis and makes a right turn. Similarly, when the input angles $\theta_{1}$ and $\theta_{2}$ satisfy $\theta_{1}<\theta_{2} \in\left(-\frac{\pi}{2}, \frac{\pi}{2}\right)$, the mechanism makes a left turn. The periodical 3D diagram on the motion space of the midpoints of the left and right feet $A_{2}$ and $A_{3}$ in the fixed coordinate system was plotted in Matlab (Fig. 8).
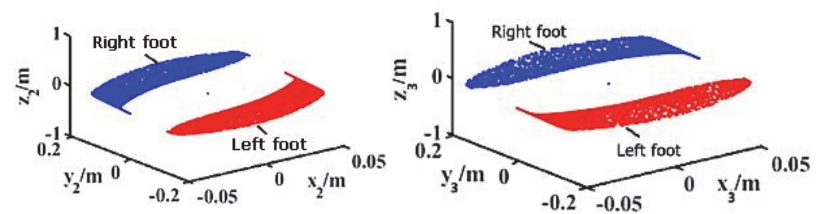

Figure 8 Periodical 3D motion space of points $A_{2}$ and $A_{3}$ : (a) $\theta_{1}>\theta_{2}$, (b) $\theta_{1}<\theta_{2}$

\subsection{Stability Analysis of Rolling Mode}

When the input angles $\theta_{1}$ and $\theta_{2}$ meet $\theta_{1}=-\theta_{2} \in\left(-\frac{\pi}{2}, \frac{\pi}{2}\right)$, the midpoints $A_{2}$ and $A_{3}$ move at the same step length along the $X$-axis and the $Z$-axis. In this case, the output rotating angles of the rod obey $\Psi_{1=} \Psi_{2}=\Psi$. Thus, the two single-loop planar 4R systems rotate synchronously, such that the mechanism rolls periodically over time. With the periodical change to input angles $\theta_{1}$ and $\theta_{2}$, the rod, the left foot and the right foot contact with the ground in turns, and the dynamic and static platforms operate alternately, turning the mechanism into the rolling mode.

The zero-moment point (ZMP) principle [14, 15] suggests that a mechanism reaches a stable state when its ZMP falls in the supporting area. Therefore, the ZMP point of our mechanism must lie in the support plane for stability.

In rolling mode, our mechanism rolls along the $X$-axis, around the $Y$-axis and periodically fluctuates along the $Z$ - 
axis. Here, only the stability along the $X$-axis is considered. The general formula of the ZMP can be expressed as [15]:

$$
x_{z m p}=\frac{\sum_{i=1}^{n} m_{i}\left(\ddot{z}_{i}+\mathrm{g}\right) x_{i}-\sum_{i=1}^{n} m_{i} \ddot{x}_{i} z_{i}-\sum_{i=1}^{n} I_{i y} \ddot{\Omega}_{i y}}{\sum_{i=1}^{n} m_{i}\left(\ddot{z}_{i}+\mathrm{g}\right)}
$$

where $x_{i}, y_{i}$ and $z_{i}$ are the centroid coordinates of each $r$ rod; $\ddot{x}_{i}, \ddot{y}_{i}$ and $\ddot{z}_{i}$ are the centroid accelerations of each rod $I_{i y}$ is the inertia moments of the rod around the $y$-axes; $\ddot{\Omega}_{i y}$ is the angular centroid accelerations of the bar around the $y$-axes, respectively; $\mathrm{g}$ is the acceleration of gravity (9.8); $m_{i}$ is the mass of each rod; $\omega$ is the angular velocity of $R_{1} R_{2}$; $\alpha$ is the angular acceleration of each rod; $m$ is the mass of each rod.

When the rod $R_{1} R_{2}$ rotates around the $Y$-axis by an angle of $\Psi$, The ZMP trajectory of the mechanism rolling along the $X$-axis can be obtained as:

$$
\begin{aligned}
& x_{z m p}=\frac{4 n \mathrm{~g}(\cos \Psi+1)-n^{2} \omega^{2} \sin \Psi \cos \Psi-n^{2} \alpha \cos ^{2} \Psi}{8 \mathrm{~g}-4 n \alpha \cos \Psi-4 n \omega^{2} \sin \Psi}- \\
& -\frac{8 n^{2} \alpha-6 n^{2} \alpha \cos \Psi-2 n^{2} \omega^{2} \sin \Psi}{8 \mathrm{~g}-4 n \alpha \cos \Psi-4 n \omega^{2} \sin \Psi}
\end{aligned}
$$

Thus, from Eq. (9) the ZMP trajectory of the mechanism along the $X$-axis hinges on such factors as rod length $n$, rotating angle $\Psi$, angular velocity $\omega$ and angular acceleration $\alpha$. Considering this, $\Psi \in\left(-\frac{\pi}{2}, 0\right)$ was selected for further analysis.

When rods $R_{1} R_{2}$ and $R_{2} R_{3}$ rotate by an angle of $\Psi$, the centroid coordinates of the mechanism can be expressed as:

$X_{G}=\frac{n+n \cos \Psi}{2} \in(0, n)$

Eq. (11) indicates that the centroid position of the mechanism always falls in the range of rod length along the $X$-axis. In this way, the stable condition of the mechanism during rolling was established, where $n=0.1 \mathrm{~m}, \omega=0 \mathrm{rad} / \mathrm{s}$ and $\alpha=0 \mathrm{rad} / \mathrm{s}^{2}$. Eqs. (10) and (11) were programmed in Matlab to output the trajectories of the ZMP and the centroid of the mechanism at the angle of $\Psi$ (Fig. 9).
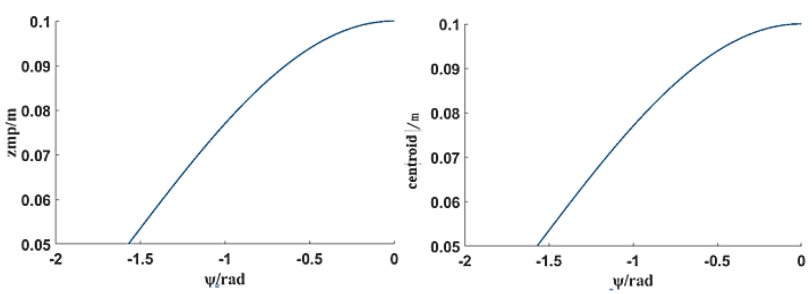

Figure 9 The trajectories of ZMP and centroid positions with changing $\Psi(n=$ $0.1 \mathrm{~m}, \omega=0 \mathrm{rad} / \mathrm{s}$ and $\alpha=0 \mathrm{rad} / \mathrm{s}^{2}$ ): (a) ZMP; (b) centroid

It can be seen that the ZMP trajectory agrees with the centroid trajectory at the rotating angle of $\Psi$ when both angular velocity and acceleration are zero. This verifies the correctness of the ZMP trajectory calculation formula.
Hence, the mechanism stability in rolling mode depends on angular velocity $\omega$ and angular acceleration $\alpha$, in addition to the main structural parameters $n$ and $p$. To identify the effect of each parameter on mechanism stability, the ZMP trajectories were simulated at constant rod length, constant angular acceleration and different angular velocities $(n=$ $0.1 \mathrm{~m}, \omega=1-21 \mathrm{rad} / \mathrm{s}$ and $\alpha=0 \mathrm{rad} / \mathrm{s}^{2}$ ).

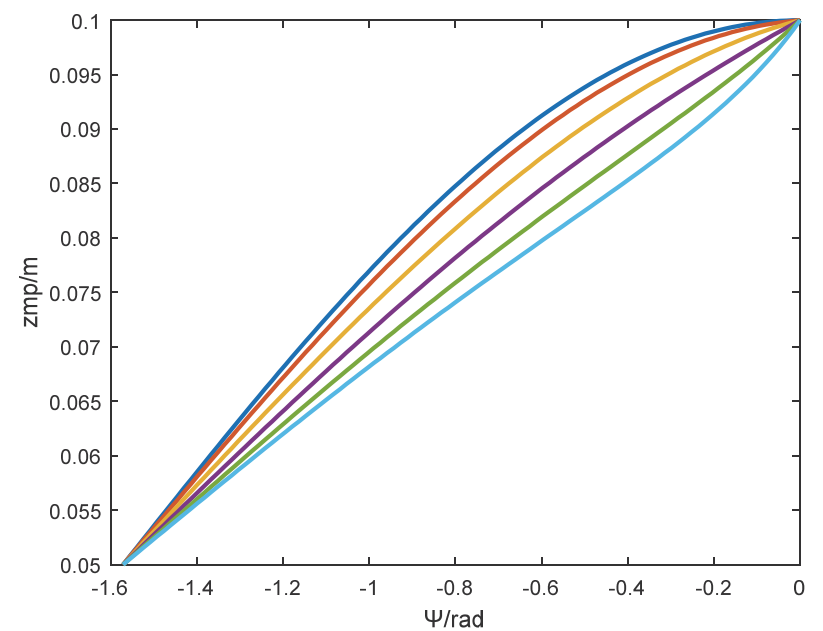

Figure 10 The trajectory of the ZMP with changing $\psi(n=0.1 \mathrm{~m}, \omega=1-21 \mathrm{rad} / \mathrm{s}$ and $\left.\alpha=0 \mathrm{rad} / \mathrm{s}^{2}\right)$

In Fig. 10, each curve represents the positions of the $\mathrm{ZMP}$ at a specific angular velocity. From top to bottom, the angular velocity is gradually on the rise, while the ZMP trajectory shifts from a convex curve to a concave curve. In the convex segment, the angular velocity is negatively correlated with the ZMP velocity and positively with mechanism stability; in the concave segment, the angular velocity is positively correlated with the ZMP velocity and negatively with mechanism stability.

Next, the ZMP trajectories were simulated at constant rod length, constant angular velocity and different angular accelerations $\left(n=0.1 \mathrm{~m}, \omega=10 \mathrm{rad} / \mathrm{s}\right.$ and $\left.\alpha=0 \mathrm{rad} / \mathrm{s}^{2}\right)$.

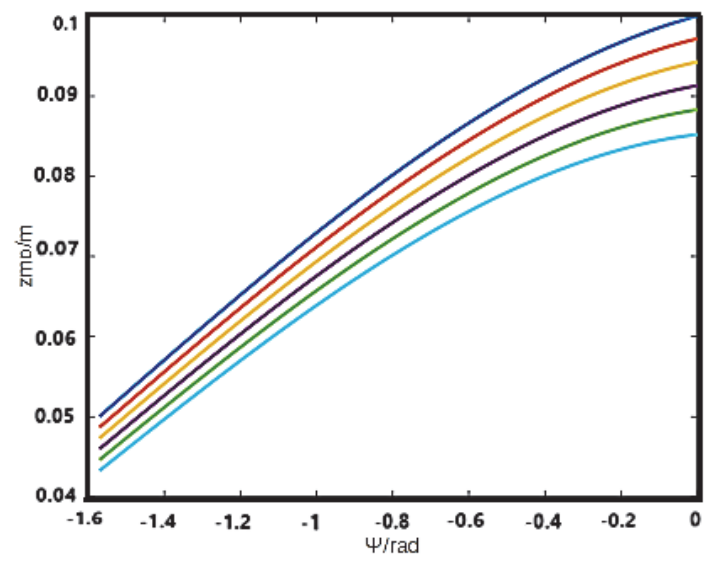

Figure 11 The trajectory of the ZMP with changing $\psi(n=0.1 \mathrm{~m}, \omega=10 \mathrm{rad} / \mathrm{s}$ and $\alpha=0 \mathrm{rad} / \mathrm{s}^{2}$ )

In Fig. 11, each curve represents the positions of the ZMP at a specific angular acceleration. From top to bottom, the angular acceleration is gradually on the rise, while the ZMP trajectory exhibits a declining trend. Under the same rod length and angular velocity, the angular acceleration is negatively correlated with the backward 
movement velocity of the ZMP. In other words, the greater the angular acceleration, the more stable the mechanism will be. However, the mechanism may turn over if the angular acceleration is too large. For instance, Fig. 12 illustrates the ZMP trajectory at $\Psi$ when the angular acceleration is as high as $\alpha=100 \mathrm{rad} / \mathrm{s}^{2}$. It is clear that the ZMP moves backward faster and faster when the angular acceleration is too large, adding to the risk of tipping of the mechanism.

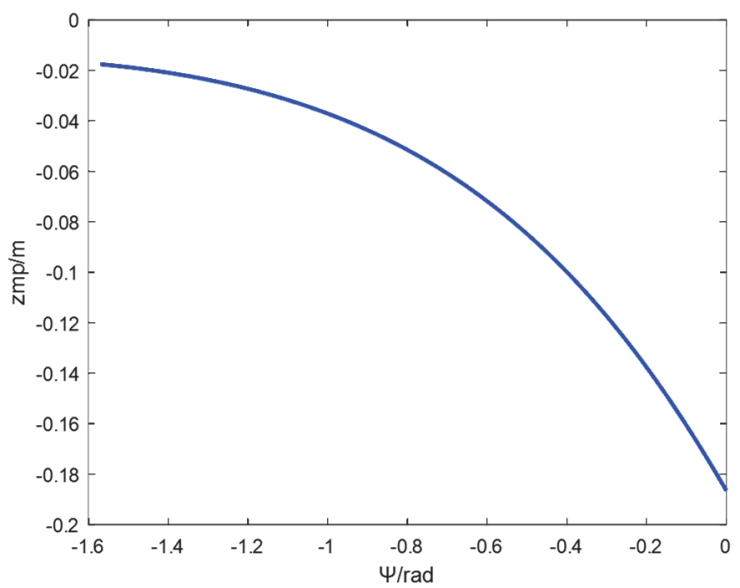

Figure 12 The trajectory of the ZMP with changing $\Psi(n=0.1 \mathrm{~m}, \omega=10 \mathrm{rad} / \mathrm{s}$ and $\alpha=100 \mathrm{rad} / \mathrm{s}^{2}$ )

To sum up, when the angular acceleration is zero and the rod length is fixed, the angular velocity is negatively correlated with the ZMP velocity and positively with mechanism stability in the convex segment of ZMP trajectory, and the angular velocity is positively correlated with the ZMP velocity and negatively with mechanism stability in the concave segment; when the angular velocity is zero and the rod length remains constant, the greater the angular acceleration, the more stable the mechanism. Of course, the mechanism may turn over if the angular acceleration is too large.

\section{STRUCTURAL OPTIMIZATION \\ 4.1 Structural Optimization of Sliding Mode}

According to the conditions of sliding mode, the mechanism will not roll over if $0 \leq y_{2}^{\prime} \leq y_{G}^{\prime} \leq y_{3}^{\prime} \leq p+\frac{2 n^{2}}{p}$. The mechanism can remain stable in the motion as long as its size parameters $n$ and $p$ satisfy the following conditions:

Let

$y_{a}=y_{G}^{\prime}-y_{2}^{\prime}$

$y_{b}=y_{3}^{\prime}-y_{G}^{\prime} \geq 0$

$y_{c}=p+\frac{2 n^{2}}{p}-y_{3}^{\prime}$

$y_{d}=y_{2}^{\prime} \geq 0$

where

$$
\begin{aligned}
& y_{2}^{\prime}=\frac{n^{2}\left(1-\cos \theta_{i}\right)}{p} \cos \theta_{i}+p- \\
& -\sqrt{p^{2}-n^{2}\left(1-\cos \theta_{i}\right)^{2}}, \quad i=1,2 \\
& y_{3}^{\prime}=\frac{n^{2}\left(1-\cos \theta_{i}\right)}{p}+p, \quad i=1,2
\end{aligned}
$$$$
y_{G}^{\prime}=\frac{\sqrt{p^{2}-h^{2}}-\frac{n^{2}\left(1-\cos \theta_{i}\right)}{p} \cos \theta_{i}}{2}, i=1,2 .
$$

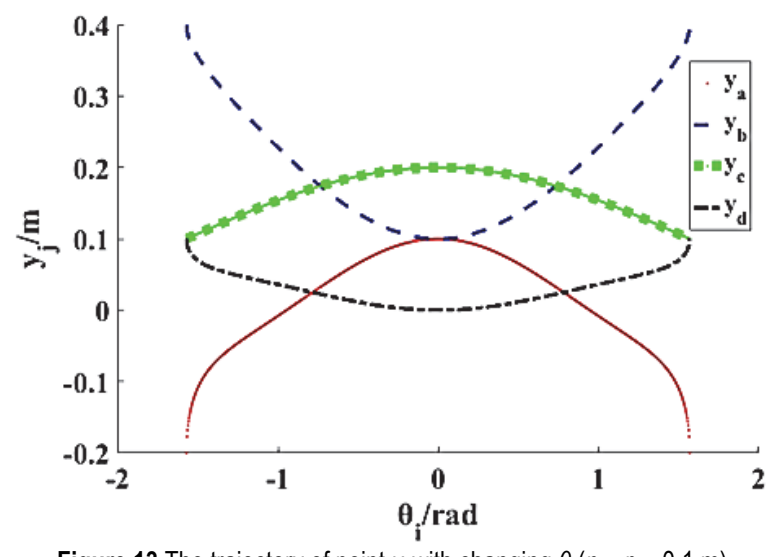

Figure 13 The trajectory of point $y_{i}$ with changing $\theta_{i}(n=p=0.1 \mathrm{~m})$

When $\frac{n}{p}=1$, Eqs. (13) to (16) were programmed in Matlab with the rod length of $n=p=0.1 \mathrm{~m}$ and $\theta_{i} \in\left(-\frac{\pi}{2}, \frac{\pi}{2}\right), i=1,2$. The trajectory of point $y_{j}>0,(j=$ $a, b, c, d)$ with the change of angle $\theta_{i}$ was obtained and shown in Fig. 13. It can be seen that, when $n=p=0.1 \mathrm{~m}$, $\frac{\theta_{i}}{\text { rad }}$ roughly falls into the range of $(-0.95,0.95)$, and the mechanism does not roll over but remains stable.
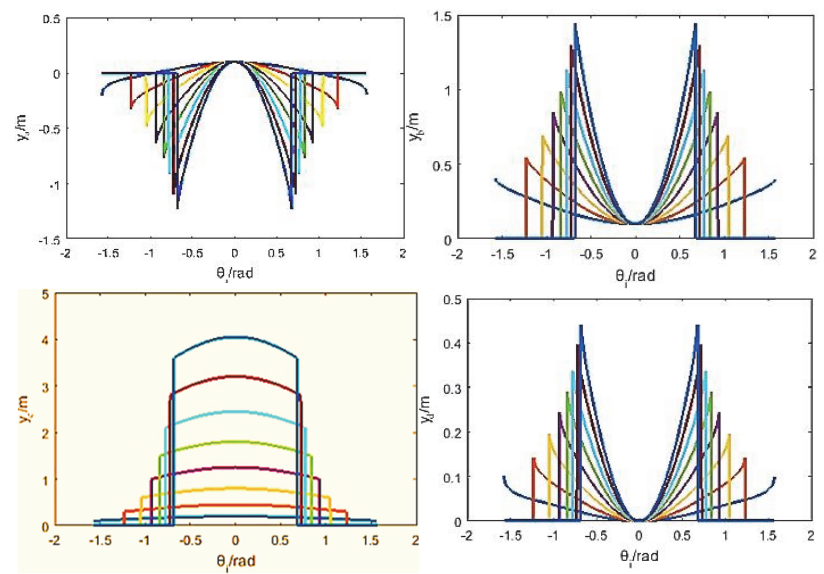

Figure 14 The trajectory of point $y_{j}$ with changing $\theta_{i}(p=0.1 \mathrm{~m}$ and $n=0.1-0.5$ m) (a) $y_{a} ;$ (b) $y_{b} ;$ (c) $y_{c} ;$ (d) $x_{d}$

When $\frac{n}{p}>1$, the trajectory of point $y_{j}$ was plotted at the rod length of $n=0.1-0.5 \mathrm{~m}$ and $p=0.1 \mathrm{~m}$ and recorded 
in Fig. 14, where the $X$-axis is the rotating angle $\theta_{i}$ and the $Y$-axis is the $y_{j}$ point position and each curve represents the $y_{j}$ point trajectory at a specific rod length. From top to bottom, the rod length is gradually on the rise. As shown in the figure, $y_{b}, y_{c}$ and $y_{d}$ all meet the requirement of $y_{j}>0$, regardless of the value of $\theta_{i}$. At the abrupt changes of the curves, the rod length $\mathrm{n}$ is negatively correlated with the range of angle $\theta_{i}$ that guarantees the mechanism stability. The mechanism will lose stability once the rotating angle exceeds $\theta_{i}$. In general, the mechanism has a negative correlation with the rod length when the rotating angle remains unchanged, and with the rotating angle when the rod length remains the same.
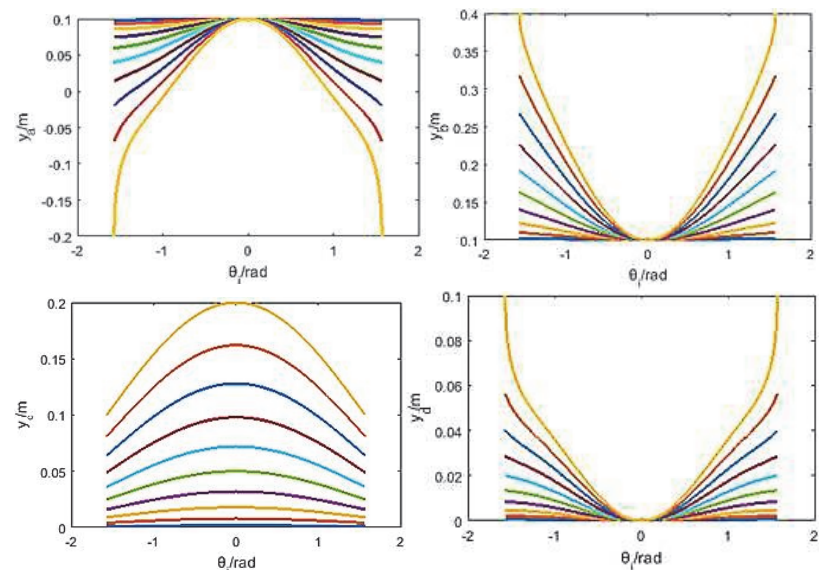

Figure 15 The trajectory of point $y_{j}$ with changing $\theta_{i}(p=0.1 \mathrm{~m}$ and $n=0.01-0.1$ m) (a) $y_{a} ;$ (b) $y_{b} ;$ (c) $y_{c}$; (d) $x_{d}$

When $\frac{n}{p}<1$, the trajectory of point $y_{j}$ was plotted at the rod length of $n=0.01-0.1 \mathrm{~m}$ and $p=0.1 \mathrm{~m}$ and recorded in Fig. 15, where each curve represents the $y_{j}$ point trajectory at a specific rod length. From top to bottom, the rod length decreases step by step. As shown in the figure, $y_{b}, y_{c}$ and $y_{d}$ all meet the requirement of $y_{j}>0$, regardless of the value of $\theta_{i}$. There is no abrupt change in the curves, that is, the mechanism remains stable in the range of $\theta_{i} \in\left(-\frac{\pi}{2}, \frac{\pi}{2}\right)$. In general, the mechanism has a negative correlation with the rod length when the rotating angle remains unchanged, and with the rotating angle when the rod length remains the same.

In summary, the greater the rod length, the smaller the range of rotating angle $\theta_{i}$ that guarantees the mechanism stability; the mechanism will lose stability once the rotating angle exceeds $\theta_{i}$; the mechanism has a negative correlation with the rod length when the rotating angle remains unchanged, and with the rotating angle when the rod length remains the same.

\subsection{Structural Optimization of Rolling Mode}

The relationship between rod length $n$ and mechanism stability was discussed in light of the ZMP trajectory Eq. (10). The trajectory of the ZMP with changing angle $\Psi\left(\Psi \in \frac{\pi}{2}, 0\right)$ was obtained through Matlab programming at the rod length of $n=0.1-0.2 \mathrm{~m}$, the angular velocity of $\omega=0 \mathrm{rad} / \mathrm{s}$ and the angular acceleration of $\alpha=0 \mathrm{rad} / \mathrm{s}^{2}$. The trajectory diagram (Fig. 16) shows a negative correlation between the rod length and the mechanism stability.

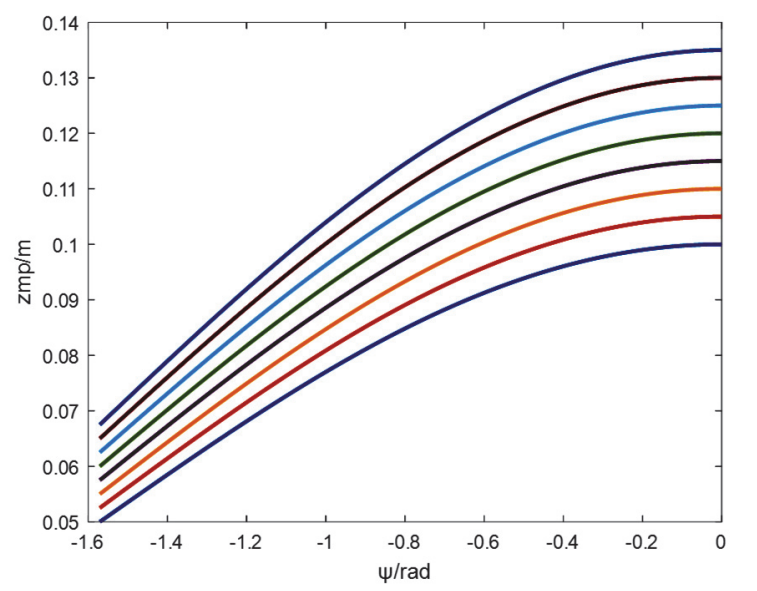

Figure 16 The trajectory of ZMP with changing angle $\Psi(n=0.1-0.2 \mathrm{~m}, \omega=0$ $\mathrm{rad} / \mathrm{s}$ and $\alpha=0 \mathrm{rad} / \mathrm{s}^{2}$ )

Through the above stability optimization, it is concluded that the mechanism is more stable at a short rod length and small rotating angle, when its width $\mathrm{p}$ is constant.

\section{SIMULATION AND PROTOTYPE TEST 5.1 Simulation 5.1.1 Simulation Analysis of Sliding Mode}

In the proposed multi-mode mobile parallel mechanism, two motors are arranged diagonally to drive two single-loop planar 4R systems separately in sliding mode. The whole mechanism moves forward due to the friction force between the ground and the two feet. To verify the analysis results, a kinematics model of the mechanism was established for the sliding mode and simulated in MSC ADAMS.

As shown in Fig. 17, the mechanism went through several kinematic phases in a period of sliding. In phases (a) to (e), the mechanism slid forward towards the right front with a small displacement along the $Y$-axis; In phases (f) to (j), the mechanism slid forward towards the left front, such that the $Y$-axis displacement was corrected. In this way, the mechanism slid in a straight line in the sliding mode.

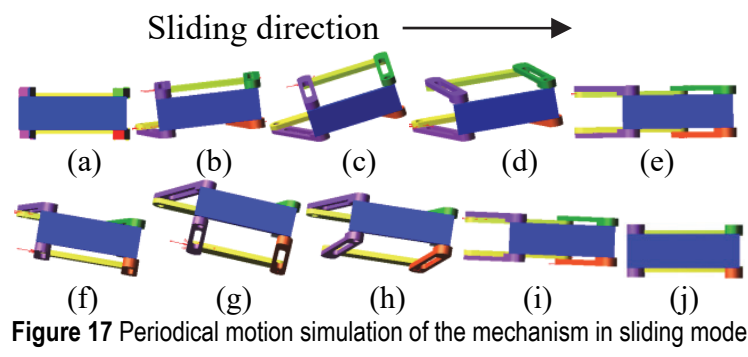

The temporal variation in displacement of point on $X$-, $Y$ - and $Z$-axes was simulated through ADAMS at and (Fig. 18). It is clear that the mechanism moves forward along the $X$-axis, with no displacement on $Z$-axis. The distance between its positions in two adjacent periods is about 40 $\mathrm{mm}$. There is a small displacement on the $Y$-axis, which 
signifies the cumulative error. Thus, the movement of the mechanism along the $Y$ - and $Z$-axes is negligible in the sliding mode.

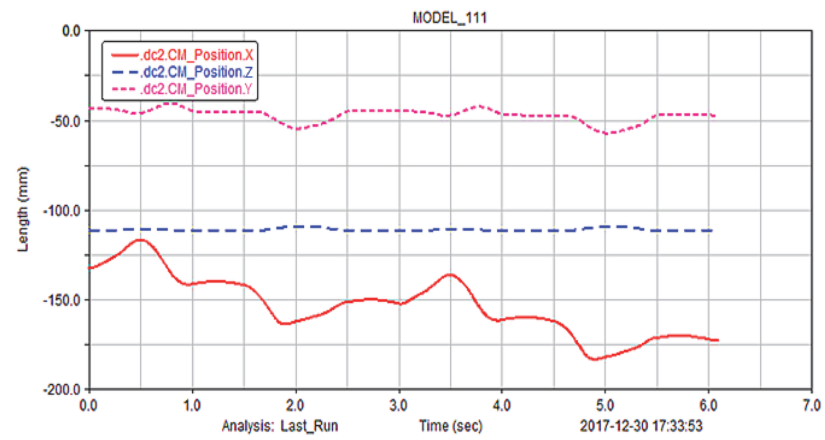

Figure 18 Temporal variation in displacement of point $A_{3}$ on $X-, Y$ - and Z-axes in sliding mode

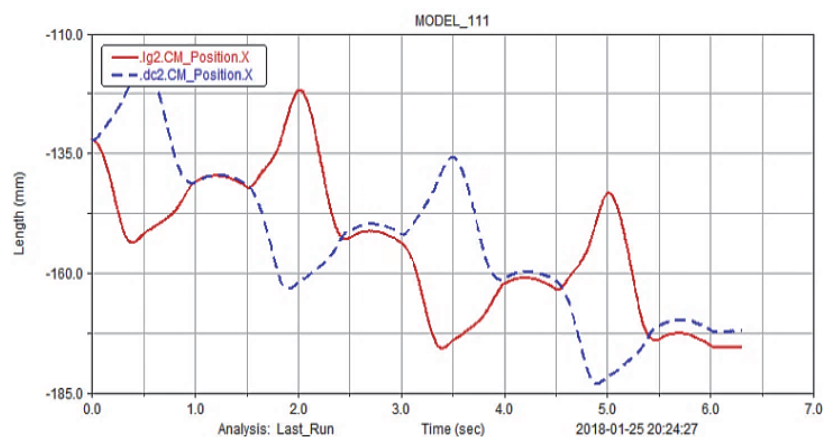

Figure 19 Temporal variation in displacement of points $A_{2}$ and $A_{3}$ on $x$-axis in sliding mode

Taking the midpoints of the left and right feet $A_{2}$ and $A_{3}$ as the typical points, the author plotted their displacements over time along the $X$-axis (Fig. 19). It can be observed that the outputted midpoints $A_{2}$ and $A_{3}$ are alternately and symmetrically distributed along the $X$-axis when the two input angles are equal $\left(\theta_{1}=\theta_{2}\right)$. The observation verifies the mathematical model and the position analysis of sliding mode.

\subsubsection{Simulation Analysis of Turning Mode}

The mechanism enters the turning mode when the two input angles are different $\left(\theta_{1}>\theta_{2}\right.$ or $\left.\theta_{1}<\theta_{2}\right)$. To verify the analysis results, a kinematics model of the mechanism was established for the turning mode and simulated in MSC ADAMS. As shown in Fig. 20, the mechanism went through several kinematic phases of turning motion within a period of time. The simulation proves that the mechanism can realize the turning mode.

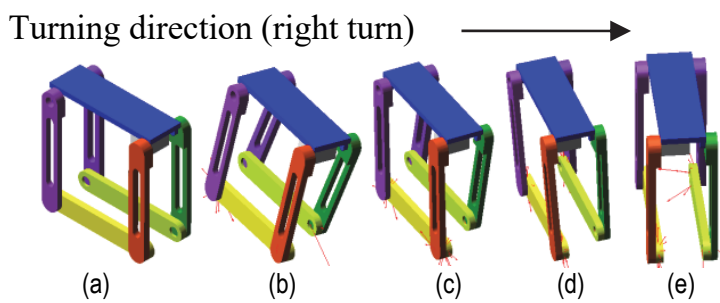

Figure 20 Periodical motion simulation of the mechanismin turning mode (right turn)

The temporal variation in displacement of point $A_{3}$ on $X$-, $Y$ - and $Z$-axes in turning mode was simulated with the same parameters as above. The simulated results (Fig. 21) reveal that the mechanism has displacement in all directions except the $Z$-axis. This means the mechanism can realize the turning along the $Z$-axis in $X O Z$ plane.

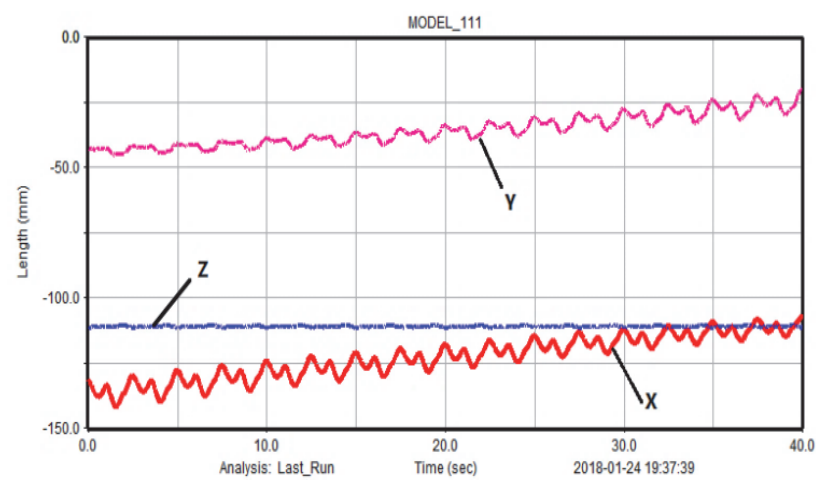

Figure 21 Temporal variation in displacement of point $A_{3}$ on $X-, Y$ - and Z-axes in turning mode (right turn)

\subsubsection{Simulation Analysis of Rolling Mode}

The mechanism enters the rolling mode when the two input angles satisfy $\theta_{1}=-\theta_{2}$. To verify the analysis results, a kinematics model of the mechanism was established for the rolling mode and simulated in MSC ADAMS. As shown in Fig. 22, the mechanism went through several kinematic phases of rolling motion within a period of time. The simulation proves that the mechanism can realize the rolling in $X O Z$ plane.

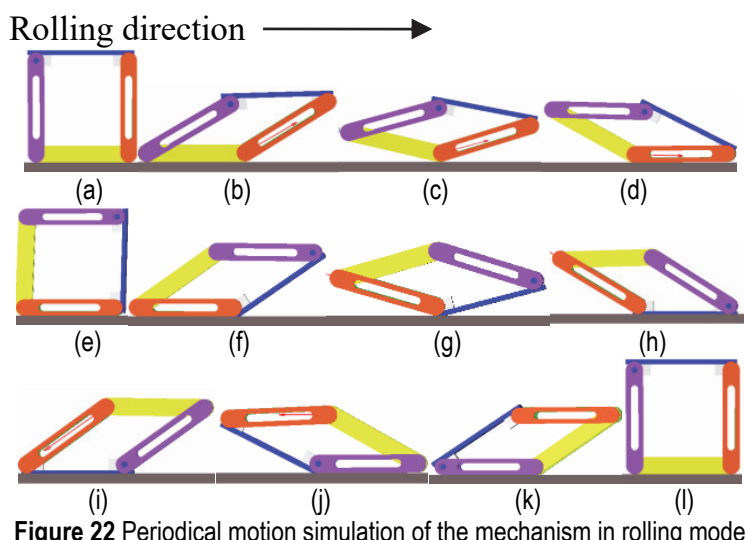

Figure 22 Periodical motion simulation of the mechanism in rolling mode

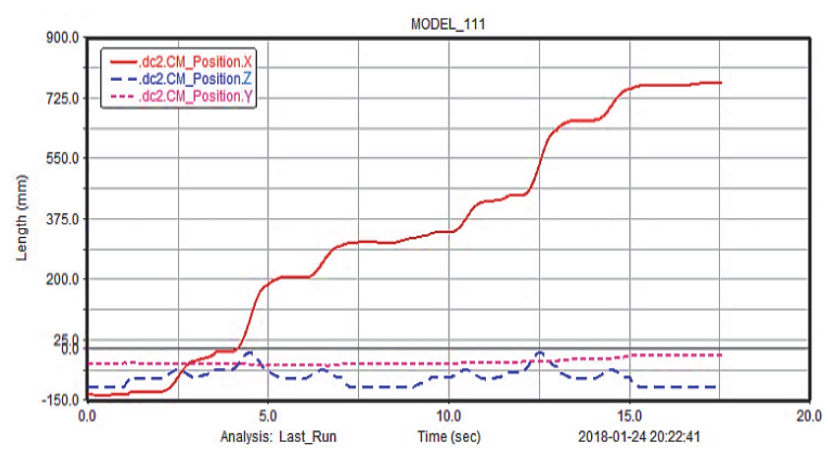

Figure 23 Temporal variation in displacement of point $A_{0}^{\prime}$ on $X$-, $Y$ - and Z-axes in rolling mode

The temporal variation in displacement of rod midpoint $A_{0}^{\prime}$ on $X$-, $Y$ - and $Z$-axes in rolling mode was simulated with the same parameters as above. According to the results in Fig. 23, the mechanism has no displacement along the $Y$-axis within a period of time, but 
moves forward along the $X$-axis. In other words, the mechanism rolls forward as a whole. Meanwhile, there are periodical changes along the $Z$-axis, a signal of periodical centroid variation of the mechanism.

To validate the kinematic simulation of the mechanism in rolling mode, the trajectory of the midpoint $A_{0}^{\prime}$ of rod $R_{2} R_{3}$ in the $X O Z$ plane was simulated in MSC ADAMS. It can be seen from the results that the simulated trajectory coincides with the trajectory of point $A_{0}^{\prime}$ in $X O Z$ plane. This further verifies the correctness of the motion simulation.

\subsection{Prototype Test}

The optimized mechanism design was made into a prototype, in which the multi-mode motion of the mechanism is driven by two motors. The motions include sliding, turning and rolling. The periodical motion phases of the prototype in each mode were recorded in Fig. 24.

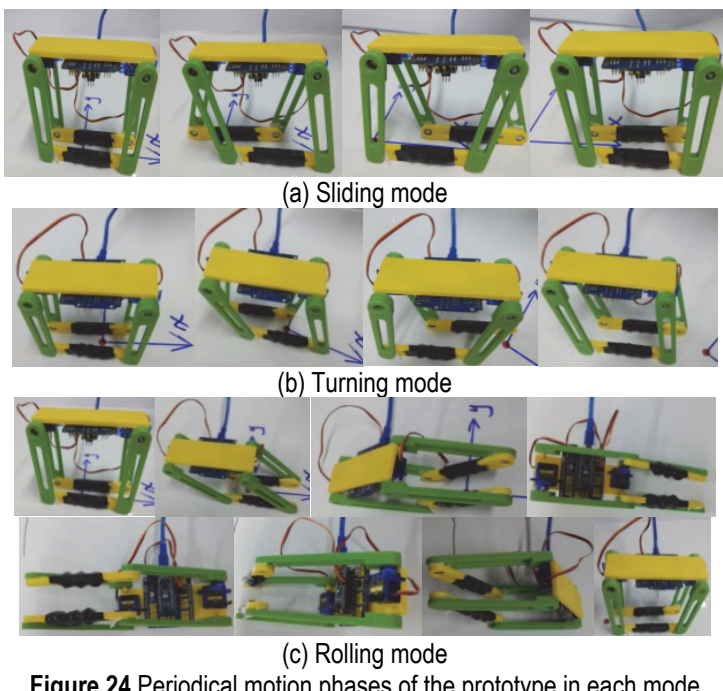

\section{CONCLUSIONS}

This paper designs a multi-mode mobile parallel mechanism capable of sliding, turning and rolling. The mechanism boasts a symmetrical structure, few DOFs and multi-mode movement, thanks to the adoption of singleloop closed-chain 4R systems. Each single-loop closedchain $4 \mathrm{R}$ system has only 1 DOF. Driven by two diagonally distributed motors, the proposed mechanism can switch freely between different modes of movement as long as the motor input angles satisfy the relationships in Tab. 1 .

Table 1 The relationship among input angle, rod output and motion mode

\begin{tabular}{|c|c|c|}
\hline input angle $\theta_{1}, \theta_{2}$ & rod output $\Psi_{1}, \Psi_{2}$ & motion mode \\
\hline$\theta_{1}=\theta_{2}$ & $\Psi_{1}=-\Psi_{2}$ & sliding mode \\
\hline$\theta_{1}>\theta_{2}$ or $\theta_{1}<\theta_{2}$ & $\Psi_{1}<\Psi_{2}$ or $\Psi_{1}>\Psi_{2}$ & turning mode \\
\hline$\theta_{1}=-\theta_{2}$ & $\Psi_{1}=\Psi_{2}$ & rolling mode \\
\hline
\end{tabular}

The author established a mathematical model of the mechanism in sliding and rolling modes, discussed the kinematics of the mechanism and optimized its stability. It is concluded that the mechanism is more stable at a short rod length and small rotating angle, when its width $\mathrm{p}$ is constant.

The research findings provide a valuable reference for similar studies on multi-mode mobile parallel mechanism, and lay the theoretical basis for the application of mobile robots in various fields. The future research will further improve the multi-mode movement of the single-loop planar 4R mechanism and enhance its applicability in actual practice. Although the stability index has been optimized in this paper, other performance, such as power consumption, moving efficiency, structure, etc., can be optimized in the subsequent work to make it more applicable. After that, friendly control systems and control algorithms can be built to realize intelligent remote control and mobile functions.

\section{REFERENCES}

[1] Hunt, K. H. (1978). Kinematic Geometry of Mechanisms. Cambridge, Cambridge University Press Books

[2] Kong, X. (2018). A variable-dof single-loop 7r spatial mechanism with five motion modes. Mechanism and Machine Theory,120, 239-249. https://doi.org/10.1016/j.mechmachtheory.2017.10.005

[3] Kong, X. \& Martin, P. ( 2015. Type synthesis and reconfiguration analysis of a class of variable-DOF singleloop mechanisms. Mechanism and Machine Theory, 85,116128. https://doi.org/10.1016/j.mechmachtheory.2014.10.011

[4] Pfurner, M., Kong, X., \& Huang, C. (2014). Complete kinematic analysis of single-loop multiple-mode 7-link mechanisms based on bennett and overconstrained rprp mechanisms. Mechanism \& Machine Theory, 73, 117-129. https://doi.org/10.1016/j.mechmachtheory.2013.10.012

[5] Zhihuai, M., Yan'An, Y., \& Xianwen, K. (2014). Biped walking robot based on a 2-upu+2-uu parallel mechanism. Chinese Journal of Mechanical Engineering, (02), 269-278. https://doi.org/10.3901/CJME.2014.02.269

[6] Tian, Y., Yao, Y. A., Ding, W., \& Xun, Z. (2016). Design and locomotion analysis of a novel deformable mobile robot with worm-like, self-crossing and rolling motion. Robotica, 34(9), 1-18. https://doi.org/10.1017/S0263574714002689

[7] Mark, Y., Shen, W. M., Behnam, S. et al. (2007). Modular SelfReconfigurable Robot Systems. IEEE Robotics and Automation Magazine, 14 (1), 43-52. https://doi.org/10.1109/MRA.2007.339623

[8] Salemi, B., Moll, M., \& Shen, W. M. (2007). SUPERBOT: A Deployable, Multi-Functional, and Modular SelfReconfigurable Robotic System. IEEE/RSJ International Conference on Intelligent Robots \& Systems, 3636-3641. https://doi.org/10.1109/IROS.2006.281719

[9] Wang, N., Fang, Y., \& Zhang, D. (2015). A spatial single loop kinematotropic mechanism used for biped/wheeled switchable robots. International Journal of Mechanics \& Materials in Design, 11(3), 287-299. https://doi.org/10.1007/s10999-014-9274-x

[10] Miao, Z., Wang, J., \& Li, B. (2017). Mobile Robot with Multiple Modes Based on 4-URU Parallel Mechanism. New Trends in Mechanism and Machine Science. Springer International Publishing, 339-407. https://doi.org/10.1007/978-3-319-44156-6_41

[11] Huang Z., Zhao, Y., \& Zhao, T. (2006) Advanced Spatial Mechanism. China, Beijing: Higher Education Press Books.

[12] Saeed, B. N. (2001). Introduction to Robotics: Analysis, Systems, Applications. Prentice Hall.

[13] Shanjun, C. \& Yuanlou, G. (2006). Application of montecarlo method in analysis of the workspace of tricept robot. Machine Tool \& Hydraulics, 11, 62-64.

[14] Vukobratovic, M, Frank, A. A., \& Juricic, D. (2008). On the stability of biped locomotion. IEEE Transactions on Biomedical Engineering, 17(1), 25-36. https://doi.org/10.1109/TBME.1970.4502681 
[15] Shigeki, T. \& Kaoru, U. (2002). Dynamic model of human walking mechanism dynamic model based on zmp principle and minimum consumption energy principle. Journal of the Japan Society for Precision Engineering.

\section{Contact information:}

Chunyan ZHANG, Associate professor

(Corresponding author)

School of Mechanical and Automotive Engineering

Shanghai University of Engineering Science, P. R. China,

Institution College of Mechanical Engineering,

Donghua University, Shanghai, P. R. China,

No. 333, Longteng Road, Songjiang District, Shanghai, P. R. China, 201620

E-mail: keke204102@163.com

\section{Mingjuan XIE, Engineer}

Engineering Technology Center

Huayu Automotive Body Components Technology (Shanghai) Co., Ltd.

P. R. China,

No. 303, Guoding Road, Yangpu District Shanghai, P. R. China, 200082

E-mail: 1658408848@qq.com

\section{Dan ZHANG, Professor}

College of Mechanical Engineering,

Donghua University, Shanghai, P. R. China

No. 2999, North Renmin Road, Songjiang District,

Shanghai, P. R. China, 201620

E-mail: dzhang99@yorku.ca 